\title{
Morphological and molecular identification of Cytospora chrysosperma causing canker disease on Prunus persica
}

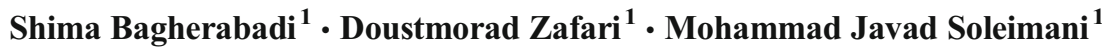

Received: 22 November 2016 / Accepted: 11 May 2017 / Published online: 24 May 2017

(C) Australasian Plant Pathology Society Inc. 2017

\begin{abstract}
Peach (Prunus persica) is one of the most valuable fruit trees around the world including Iran. During spring and summer 2016, severe branch dieback and canker was observed on peach trees in Hamadan province, Iran. The cankered peach twigs contained fruiting bodies of the fungus. It was estimated that $58 \%$ of peach trees were severely damaged by this disease. A fungus was consistently isolated from infected tissues. Morphological and cultural characteristics, as well as the sequences of the ITS and $\beta$-tubulin regions and pathogenicity tests, showed that the causal agent of disease was Cytospora chrysosperma. It was already reported that C. chrysosperma caused severe losses on a wide range of plant hosts worldwide. However, to our knowledge, this is the first report of C. chrysosperma on P. persica in the world and Iran.
\end{abstract}

Keywords Its $\cdot \beta$-tubulin $\cdot$ Cytospora chrysosperma $\cdot$ Prunus persica

Peach (Prunus persica) is an economically important deciduous tree belonging to the Rosaceae family producing 20 million tons of fruit per year worldwide. It is one of the most important fruit trees around the world including Iran. The peach orchards with dieback and canker symptoms were observed during spring and summer 2016, in Hamadan province of Iran. The symptoms included wood lesions and cankers with fruiting bodies from fungus (Fig. 1a). 15 orchards were assessed from five different regions of Hamadan province and 80 trees out

Doustmorad Zafari

Zafari_d@yahoo.com

1 Department of Plant Protection, Faculty of Agriculture, University of Bu-Ali Sina, Hamadan, Iran of 137 inspected trees displayed typical symptoms of disease. It was estimated that $58 \%$ of peach trees were severely damaged by this disease. In order to identify the causal agent of the disease, a total of 80 diseased twigs were collected from symptomatic trees. Small pieces, approximately $0.5 \mathrm{~cm}^{3}$, were cut from the edge of diseased and healthy tissues and sterilised in $70 \%$ ethanol for $1 \mathrm{~min}$, followed by washing with sterile distilled water and dried on sterile filter paper. The plant segments were then placed on the surface of Potato Dextrose Agar (PDA) medium and incubated at $25{ }^{\circ} \mathrm{C}$ in the darkness. Purification was done from three-day-old isolates by the mycelium tip culture technique and also in some cases, the single-spore technique was made using Adams et al. (2006) method. The cultures were preserved on PDA in $2 \mathrm{ml}$ microtube slants at $4{ }^{\circ} \mathrm{C}$ in the Culture Collection of the Bu-Ali Sina University of Iran and a culture of C. chrysosperma (CYH1) isolate was also deposited in the Iranian Research Institute of Plant Protection Culture Collection (IRAN 2642C).

The morphological characteristics were determined based on pure cultures grown on PDA. Conidiomata were examined on infected twigs, the structure and size of fruiting bodies, presence or absence of a conceptacle, and size and shape of spores also were recorded. Dilutions were performed with the spore masses obtained from fungus fruiting bodies, and drops of suspensions were placed on microscope slides. More than 30 fruiting bodies were sectioned and in total 50 spores derived from different fruiting bodies were selected randomly to measure their lengths and widths using a Leica light microscope. Cultural characteristics of isolates grown on PDA in the darkness at $25{ }^{\circ} \mathrm{C}$ were recorded. These included colony characters and pigment production at 7,14 , and 30-days after incubation. 
Fig. 1 Cytospora chrysosperma. (a) wood lesions and canker with fungal fruiting bodies on naturally infected peach shoots; (b) wood necrosis on artificially inoculated peach shoots

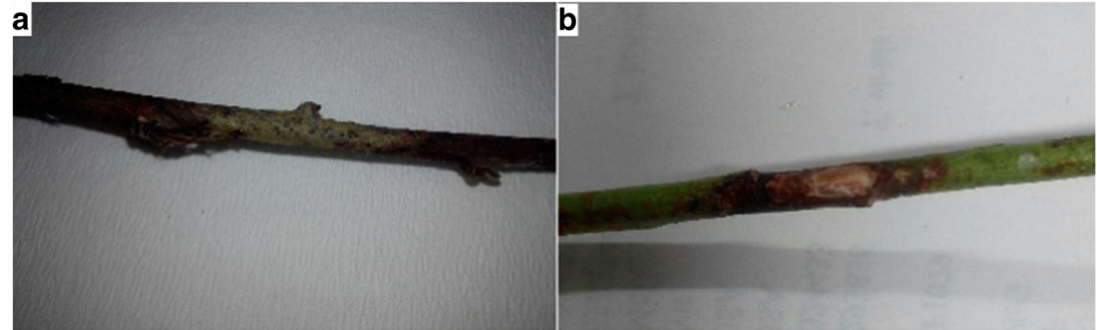

Colony color on the surface initially was white and then changed moderately to intense yellow or moderate orange yellow (Fig. 2).

Teleomorph stage was not observed. Conidiomata immersed in the bark, usually rather flat, erumpent, labyrinthine cytosporoid, with labyrinthine chambers, circular to ovoid, 1$2 \mathrm{~mm}$ in diam, disc gray to black, nearly flat, circular to ovoid, 0.14-0.49 $\mathrm{mm}$ in diam., with one ostiole per disc. Conidiomata formation was sparse, appearing after 14 days but they formed abundantly after 30 days on PDA medium. Locules were multi-chambered with dark walls. Conidiophores were hyaline, divided into two types, the first type branched and septate with 15-30 $\mu \mathrm{m}$ length, second type long, unbranched and aseptate or rarely septate. Conidia hyaline, allantoid, aseptate, (3.5-) 4(-4.5) $\times 1 \mu \mathrm{m}$, oozing out as an orange mucilaginous mass (Fig. 3). The cultural and morphological characteristics of our isolates were in agreement with the description for C. chrysosperma (Adams et al. 2006).

To confirm the morphological identification, isolate CYH1 (IRAN 2642C) was selected as a representative for molecular analysis. Genomic DNA was extracted from mycelium of pure culture grown on PDB (Potato Dextrose Broth) at $25^{\circ} \mathrm{C}$ after 7 days as described by Sharma et al. (2002). The ITS region was amplified using primers ITS1 and ITS4 (White et al. 1990), and additional $\beta$-tubulin region was amplified using primers BT2A and BT2B to improve species delineation within the genus Cytospora (Glass and Donaldson 1995). Partial DNA of a representative fungal isolate CYH1 (IRAN 2642C) was amplified using ITS1 and ITS4 primer pairs for ITS regions and BT2A and BT2B for $\beta$-tubulin gene region. The PCR reactions were performed in a TC-512 (Techne) thermocycler in a total volume of $25 \mu \mathrm{l}$. The PCR reaction contained $10 \mathrm{ng}$ of genomic DNA, $1 \mu \mathrm{M}$ of each primer, $0.2 \mathrm{mM}$ of dNTPs (CinnaGen, Iran), 1X PCR buffer, $2.5 \mathrm{mM} \mathrm{MgCl}_{2}$ and $1 \mathrm{U}$ Taq DNA polymerase (CinnaGen, Iran). PCR conditions for ITS and $\beta$-tubulin included an initial denaturation step at $94{ }^{\circ} \mathrm{C}$ for $5 \mathrm{~min}$, followed by 35 cycles at $94{ }^{\circ} \mathrm{C}$ for $1 \mathrm{~min}, 58^{\circ} \mathrm{C}$ (ITS) and $55^{\circ} \mathrm{C}$ ( $\beta$-tubulin) for $1 \mathrm{~min}$, and $72{ }^{\circ} \mathrm{C}$ for $1 \mathrm{~min}$, with a final extension at $72^{\circ} \mathrm{C}$ for $10 \mathrm{~min}$. The PCR products were purified using AccuPrep PCR Purification Kit and sequencing was performed either in the forward ( $\beta$-tubulin) or reverse (ITS) direction by Bioneer (South Korea). Raw sequences were edited in the BioEdit v. 7.0.5.2 (Hall 1999). Homology searches were performed using the BLAST program of the National Center for Biotechnology Information (NCBI). BLAST search analyses showed that the sequence data for two loci had the highest similarity to Cytospora chrysosperma. The sequences were deposited in GenBank (ITS: KX863715 and $\beta$-tubulin: KX961725).

Sequences were aligned in the CLUSTAL-X v. 1.81 (Thompson et al. 1997) and the Neighbour-Joining (NJ) analysis was performed on the combined ITS and $\beta$-tubulin sequences of the isolate CYH1 (IRAN 2642C) from peach, C. chrysosperma from other hosts and sequences of species closely related to Cytospora from GenBank in MEGA v. 7 (Kumar et al. 2016) with the Kimura-2-parameter distance model (Kimura 1980). Clade stability was assessed using 1000 bootstrap replications (Felsenstein 1985). Diaporthe eres was used as the outgroup in the phylogenetic analyses (Arzanlou and Narmani 2015). The Neighbour-Joining (NJ) analysis showed that isolate CYH1 (IRAN 2642C), obtained from the cankered peach twigs, clustered with authentic isolates of C. chrysosperma (Fig. 4).
Fig. 2 Cytospora chrysosperma. (a-c) colony morphology after 7 , 14 and 30 days of growth on PDA
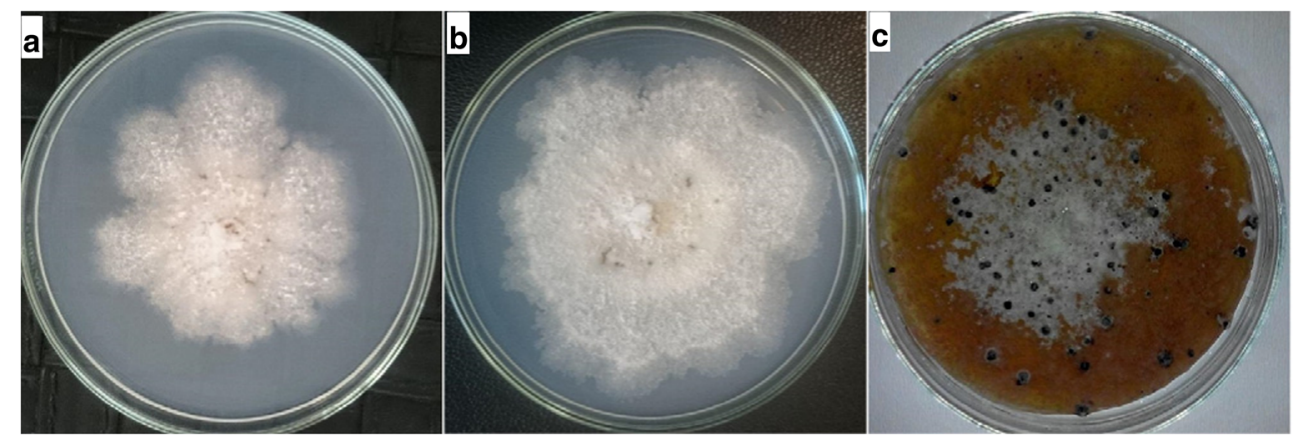
Fig. 3 Cytospora chrysosperma. (a) cross section through conidiomata; (b) ostiole, (c) conidiophores; (d) conidia. Bars $=10 \mu \mathrm{m}$
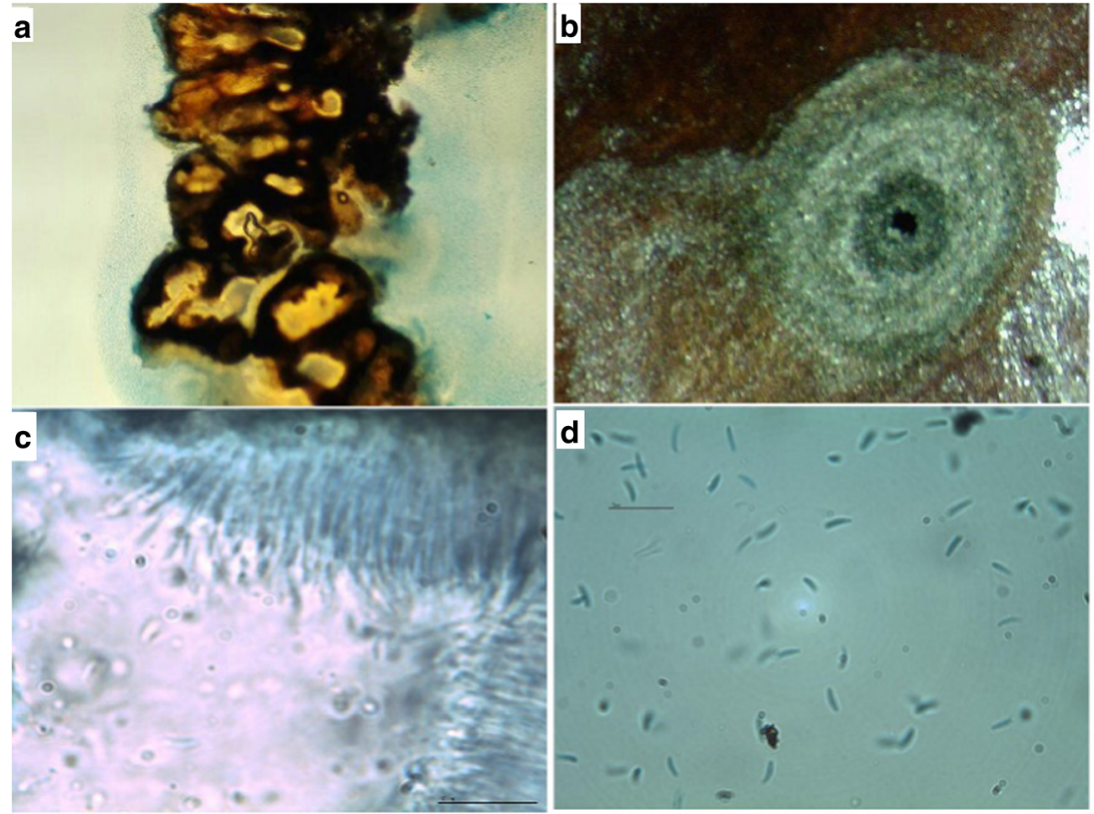

Pathogenicity tests were performed on excised shoots in laboratory conditions as described by Arzanlou and Narmani (2015). In total, 15 healthy shoots a year-old were cut from trees. The leaves were removed and shoots were surfacesterilised with $70 \%$ ethanol. For inoculation, shoots were wounded $10 \mathrm{~cm}$ above the first internode by removing the cortex using a sterile $5 \mathrm{~mm}$ diameter metal cork borer. A mycelial plug ( $5 \mathrm{~mm}$ diameter) obtained from the margin of a seven-day-old fungal colony CYH1 (IRAN 2642C) was placed in the wound. The wound was wrapped with Parafilm and for the control shoot, a plug of clean PDA was placed in the wound. Inoculated shoots were placed in a plastic container containing moistened filter papers to keep the relative humidity high. The shoots in the containers were kept in laboratory conditions at $25^{\circ} \mathrm{C}$ with natural daylight. The shoots were examined after three weeks to explore the progress of the disease. Longitudinal and cross sections were made from above and below the inoculation point. Wood necrosis was evident in the inoculated shoots (Fig. 1 b). No visible symptoms were observed on control shoots, whereas C. chrysosperma was reisolated from the edge of the disease tissue on inoculated shoots. According to the results of the pathogenicity test, culture appearance, morphological characteristics and molecular analyses of the isolated fungus, the causal agent of the canker disease of $P$. persica was identified as C. chrysosperma.

C. chrysosperma has a broad host range and is widely spread in Asia, North and South America, Africa and Oceania (Adams et al. 2006). Disease caused by C. chrysosperma was previously reported on a broad range of hosts in the world. The potential of Cytospora group to cause yield losses in economically important crops warrants further study on these fungal pathogens. Since peach is an important tree and this pathogen leads to major damage, better
Fig. 4 Single Neighbor-Joining tree depicting the phylogenetic relationship between C. chrysosperma from peach CYH1 (IRAN 2642C), C. chrysosperma from diverse hosts and other Cytospora and Leucostoma species inferred from combined ITS and $\beta$-tubulin sequences. Bootstrap values $>50 \%$ from a maximum likelihood search are indicated in bold

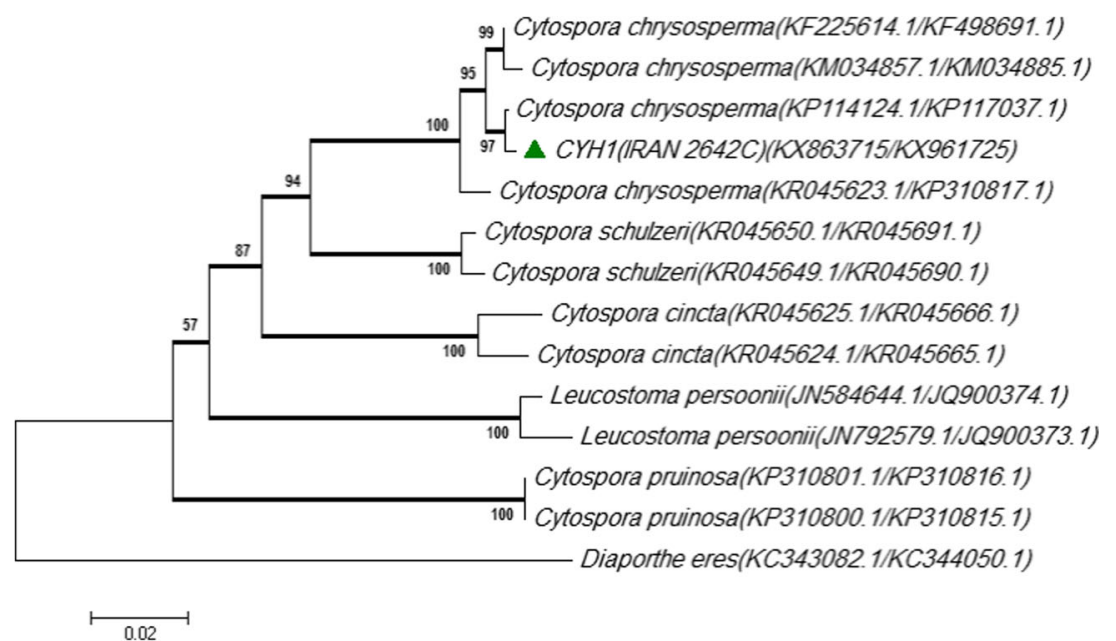


understanding of this destructive disease would lead to the establishment of better disease control strategies. It seems outbreak of canker by C. chrysosperma on peach trees was due to wounds caused by severe hail precipitation in spring 2016 in Hamadan province. To our knowledge, this is the first report of canker disease caused by $C$. chrysosperma on $P$. persica in the world and Iran.

Acknowledgements The authors would like to thank the Research Deputy of the University of Bu-Ali Sina, Hamadan, Iran for financial support.

\section{References}

Adams GC, Roux J, Wingfield MJ (2006) Cytospora species (Ascomycota, Diaporthales, Valsaceae): introduced and native pathogens of trees in South Africa. Australas Plant Pathol 35:521-548. doi:10.1071/AP06058

Arzanlou M, Narmani A (2015) ITS sequence data and morphology differentiate Cytospora chrysosperma associated with trunk disease of grapevine in northern Iran. J Plant Prot Res 55:117-125
Glass NL, Donaldson GC (1995) Development of primer sets designed for use with the PCR to amplify conserved genes from filamentous ascomycetes. Appl Environ Microbiol 61:1323-1330

Hall TA (1999) BioEdit: a user friendly biological sequence alignment editor and analysis program for windows 95/98/ NT. Nucleic Acids Symp Ser 41:95-98

Kimura M (1980) A simple method for estimating evolutionary rates of base substitutions through comparative studies of nucleotide sequences. J Mol Evol 16:111-120. doi:10.1007/BF01731581

Kumar S, Stecher G, Tamura K (2016) MEGA7: molecular evolutionary genetics analysis version 7.0 for bigger datasets. Mol Biol Evol 33: 1870-1874. doi:10.1093/molbev/msw054

Sharma AD, Prabhjot KG, Prabhjot S (2002) DNA isolation from dry and fresh samples of polysaccharide - rich plants. Plant Mol Biol Rep 20:415a-415f. doi:10.1007/BF02772129

Thompson JD, Gibson TJ, Plewniak F, Jeanmougin F, Higgins DG (1997) The Clustal-X windows interface: flexible strategies for multiple sequence alignment aided by quality analysis tools. Nucleic Acids Res 25:4876-4882. doi:10.1093/nar/ 25.24.4876

White TJ, Bruns T, Lee S, Taylor J (1990) Amplification and direct sequencing of fungal ribosomal RNA genes for phylogenetics. In: MA Innis, DH Gelfand, JJ Sninsky, TJ White (eds) PCR protocols: a guide to methods and applications. pp. 315-322. Academic Press, San Diego. doi: 10.1016/B978-0-12-372180-8.50042-1 